\title{
The Band-Importance Function for the Korean Standard Sentence Lists for Adults
}

\author{
In-Ki Jin ${ }^{1}$, Junghak Lee ${ }^{2}$, Kyoungwon Lee ${ }^{2}$, Jinsook Kim, \\ Dongwook Kim ${ }^{3}$, Junil Sohn ${ }^{3}$, and Kyung-Ju Lee ${ }^{4}$ \\ ${ }^{1}$ Division of Speech Pathology and Audiology, Research Institute of Audiology and Speech Pathology, College of Natural Sciences, \\ Hallym University, Chuncheon, \\ ${ }^{2}$ Department of Audiology, Hallym University of Graduate Studies, Seoul, \\ ${ }^{3}$ Samsung Electronics, Mobile Communications Business, Yongin, \\ ${ }^{4}$ Department of Speech Pathology and Audiology, Graduate School, Hallym University, Chuncheon, Korea
}

$\begin{array}{ll}\text { Received } & \text { February 19, 2016 } \\ \text { Revised } & \text { March 17, 2016 } \\ \text { Accepted } & \text { April 11, 2016 }\end{array}$

Address for correspondence Junghak Lee, FAAA, BCA, PhD Department of Audiology, Hallym University of Graduate Studies, 405 Yeoksam-ro, Gangnam-gu, Seoul 06198, Korea

Tel $+82-2-2051-4950$

Fax +82-2-3453-6618

E-mail leejh@hallym.ac.kr
Background and Objectives: The band-importance function (BIF) refers to a value characterizing the relative importance of different frequencies to speech intelligibility. The purpose of this study was to derive the BIF for the Korean standard sentence lists for adults (KSSL-A). Subjects and Methods: In this study, sentences from the KS-SL-A were used as the speech material. Twenty-six normal-hearing Korean listeners participated and intelligibility scores in 8 filters with 3 signal-to-noise ratio conditions were obtained. Based on the intelligibility score percentages, the BIF for the KS-SL-A was derived by using an established protocol. Results: Band-importance weights varied across frequency bands. The most important frequency region was around $316 \mathrm{~Hz}(20.0 \%)$, and the importance of the frequency bands below the center frequency (CF) of $1,778 \mathrm{~Hz}$ was $59.6 \%$. Therefore, low frequencies below the CF of $1,778 \mathrm{~Hz}$ were more important than high frequencies above the CF of $1,778 \mathrm{~Hz}$. Conclusions: The BIF for KS-SL-A could be applied towards developing a hearing aid fitting formulae for Korean listeners.

J Audiol Otol 2016;20(2):80-84

\section{Introduction}

The band-importance function (BIF) refers to a value characterizing the relative importance of different frequencies to speech intelligibility [1,2]. Each frequency band's importance weight is determined by a value between 0.0 and 1.0 . An importance function of 1.0 corresponds to a degree of $100 \%$ importance. The BIF has various significant applications in the field of hearing sciences [1-3] and is an important component of the Speech Intelligibility Index (SII), a model to predict speech intelligibility [1]. When the SII predicts a person's ability to understand speech, the predicted value is calculated based on the dynamic range of the speech, the BIF for the speech, and the person's hearing thresholds. The BIF

This is an Open Access article distributed under the terms of the Creative Commons Attribution Non-Commercial License (http://creativecommons.org/licenses/by-nc/3.0/) which permits unrestricted non-commercial use, distribution, and reproduction in any medium, provided the original work is properly cited. can also be used to determine frequency-gain response for the hearing aid fitting formula [3]. National Acoustic Laboratories' non-linear fitting procedure version 1 was developed based on the BIF and the dynamic range of speech when determining frequency-gain characteristics [3].

The BIF has been shown to vary between languages. Wong, et al. [4] derived the BIF for Cantonese sentences. The authors reported that low-frequency information was more important for understanding Cantonese speech when compared to English. In the case of Cantonese sentences, the most important frequencies were around $1,600 \mathrm{~Hz}$ and the weighting factor of band-importance for frequencies from 0 to $355 \mathrm{~Hz}$ was $13.6 \%$ in one-third octave bands. In the case of English sentences, however, the most important frequencies were around $2,000 \mathrm{~Hz}$, and the weighting factor of band importance for frequencies from 100 to $400 \mathrm{~Hz}$ was $4.63 \%$ in 9 frequency bands [5]. The results from this study suggest that different languages may have different BIFs. 
Jin, et al. [6] obtained the BIFs by using Korean hearingin-noise test (K-HINT) sentences [7]. They reported that the BIF for Korean sentences was different from that for English sentences. Specifically, low frequency bands below the center frequency $(\mathrm{CF})$ of $250 \mathrm{~Hz}$ were more important in the Korean BIF than in English BIF. For example, the band-importance weight for Korean at the CF of $250 \mathrm{~Hz}$ was $8.4 \%$, but the weight for English at the same CF was $2.65 \%$. Thus, the authors [6] concluded that the Korean BIF may be different from other languages, which have different acoustic and linguistic characteristics.

The BIF can be influenced by speech stimuli from the same language. The American National Standard Institute reported that BIFs for the Central Institute for the Deaf auditory test (CID-22) [8] and the Northwestern University auditory test number 6 (NU-6) [9] were different even if the stimulus types for both were similar [1]. For example, bandimportance weights for the CID-22 at the CFs of $150 \mathrm{~Hz}, 250$ $\mathrm{Hz}$, and $350 \mathrm{~Hz}$ were $5.07 \%, 6.77 \%$, and $6.41 \%$, respectively; however, band-importance weights for the NU-6 at the same CFs were $2.34 \%, 3.68 \%$, and $5.20 \%$, respectively. Not only did these three frequency bands differ but other frequency bands also showed different importance weights between the CID-22 words and the NU-6 words [1]. The CID-22 consisted of 200 words and each list included 50 words [8]. The CID-22 words were developed based on phonetically balanced monosyllabic words (e.g., 'wood', 'where', and 'chin') [8]. The NU-6 test was comprised of four lists of 50 words, which were developed based on phonemically balanced monosyllables (e.g., 'back', 'late', and 'such') [9]. Although both sets of test words were developed using different rationales, the stimulus type was similar. These results suggest that the BIF can be dependent on speech materials, even when they originate from the same language.

The present study was designed to derive the BIF for the Korean standard sentence lists for adults (KS-SL-A) [10]. The KS-SL-A was developed for the sentence recognition test, which was used to evaluate listening skills in everyday life. Each list comprised of 10 sentences with 40 key words that were phonetically balanced across the lists. There were a total of 8 lists. The KS-SL-A was chosen based on the selection criteria for vocabulary and sentence structures of the CID everyday sentence test [11]. Although the BIF based on the KHINT sentences has already been derived, it is also important to derive the BIF for the KS-SL-A [6] since the KS-SL-A and the K-HINT sentences were developed for different purposes. The K-HINT sentences were developed to test speech perception in a noisy environment, while the KS-SL-A were developed to test speech recognition in a quiet environment
$[7,8]$.

The purpose of this study was to derive the BIF for the KSSL-A. The results from this study provide information on the relationship between a standardized Korean sentence stimuli and the BIF. In addition, the BIF for the KS-SL-A could serve as an important tool for the development of a hearing aid fitting formula for Korean listeners.

\section{Subjects and Methods}

\section{Participants}

Twenty-six normal-hearing Korean listeners participated in this study. There were 13 male and 13 female participants (19-26 years). Their auditory thresholds were a $20 \mathrm{~dB}$ HL or better with octave frequencies from $250 \mathrm{~Hz}$ to $8,000 \mathrm{~Hz}$. All participants had normal middle ear functions.

\section{Stimuli}

Sentences of the KS-SL-A were used in the study as stimuli [10]. A total of 80 sentences were used, with 8 sets of 10 sentences per set. Each test set comprised 40 keywords. Sentences were mixed with speech-shaped noises, which were matched to the spectrum of the sentences to create signals that varied in SNR. Three SNR conditions were used (-5, 0, $+5 \mathrm{~dB}$ SNR). The noisy sentences were adjusted to have a level of $45 \mathrm{~dB}$ HL [12]. The noisy speech sentences were then filtered through both low- and high-pass filters (224, 447, 891, 1,413, 2,239, 3,548, 5,623, and 11,000 Hz) using Cooledit 2.0 (ADOBE systems, San Jose, CA, USA). Band widths were selected at regular intervals in $1 / 3$ octave band cutoff frequencies [13]. We used linear-phase finite impulse response filters with a rejection slope of $96 \mathrm{~dB} /$ octave at the desired cutoff frequencies.

\section{Equipment}

To generate the filtered stimuli, an audio program (Cooledit 2.0, Adobe Systems, San Jose, CA, USA) was used. The stimuli were routed to an audiometer (GSI61; Grason-Stadler, Eden Prairie, MN, USA) and presented to the participant's right ear through a headphone (TDH-50P; Telephonics Corporation, Farmingdale, NY, USA).

\section{Procedure}

To derive the BIF, intelligibility data were obtained using various filters with varying SNR conditions $[6,13]$. Participants listened to the speech stimuli through headphones. The task was to listen to a sentence and repeat back as much of the sentence as possible. Subsequently, intelligibility (percent keywords correct) was measured in various filtering (8 low- 
and 8 high-pass filters) with noise conditions ( $3 \mathrm{~dB}$ SNRs). Since the stimulus set included 80 sentences, each listener participated in a subset (16 conditions) of the total number of conditions (16 filters $\times 3 \mathrm{SNRs}=48$ conditions $)$ in a randomized order. Each participant took about two hours to complete the testing and the break was given every 30 minutes.

\section{Derivation of the BIF}

We used an established protocol [13] to derive the BIF. Briefly, the established protocol used an iteration technique exploiting the relationship between the BIF and the SII. Relative SII values were determined from percent correct scores. BIF values were then derived from the relative SII values.

First, the relationship between the percent correct scores and the relative SII values were determined. The initial point for the relationship was determined by plotting the percent correct scores as a function of band-frequency at the highest SNR (+5 dB SNR) used in the present study. One curve represented the data where the high frequencies were removed using a low-pass filter and the other curve represented the data where the low frequencies were removed using a highpass filter. The intersection of these two curve was considered the initial point in a series of graphical processes and curve interpolations for data at different SNRs, generating the constant values in Equation 1 [Eq. (1)]:

$$
S=\left(1-10 \frac{P A}{Q}\right)^{N}
$$

In Eq. (1), which was originally proposed by Fletcher and Galt [14], $S$ is the percent correct score, $A$ is the SII value, $P$ is a value for the measure of the talker's and listener's proficiency, and $Q$ and $N$ are fitting constants. The proficiency value, $P$, was assumed to be 1 because all participants and talkers had normal hearing. The BIF was determined using the computed values of fitting constants through the inverse of Eq. (1). Using the inverse of Eq. (1), all percent correct scores were transformed into associated SII values and the cumulative values were converted to separate values. The separate SII values for low- and high-pass filters were averaged, then the values for the same cutoff frequency band at the different SNRs were averaged. After expanding the averaged values to a 0.0 to 1.0 , the final BIF values for each frequency band were determined. Kim and Jin [15] provided a more detailed description for the BIF protocol.

\section{Results}

Mean intelligibility scores in 8 filtering and 3 SNR conditions for both low- and high-pass filters are shown in Fig. 1,
2. Overall, the average intelligibility scores increased as audible frequency band and SNR increased. At the highest SNR (+5 dB) with low-pass filtering, the mean intelligibility scores increased from $0.00 \%$ to $98.83 \%$ as cut-off frequencies increased from $224 \mathrm{~Hz}$ to $11,000 \mathrm{~Hz}$. In contrast, mean intelligibility scores in high-pass filtered conditions decreased from $99.77 \%$ to $0.00 \%$ as cut-off frequencies varied from 224 $\mathrm{Hz}$ to $11,000 \mathrm{~Hz}$. At the lowest SNR (-5 dB) with low-pass filtering, the mean intelligibility scores increased from $0.00 \%$ to $69.33 \%$ as cutoff frequencies increased from $224 \mathrm{~Hz}$ to $11,000 \mathrm{~Hz}$. In contrast, mean intelligibility scores in highpass filtered conditions decreased from $65.83 \%$ to $0.00 \%$ as cutoff frequencies varied from $224 \mathrm{~Hz}$ to $11,000 \mathrm{~Hz}$.

The BIF for KS-SL-A that was derived using the established protocol is shown in Table 1. In Eq. (1), $Q$ and $N$ values to derive the best-fit BIF were 0.4402 and 6.076, respectively. The $\mathrm{R}^{2}$ value was 0.9963 indicating a reliable relationship be-

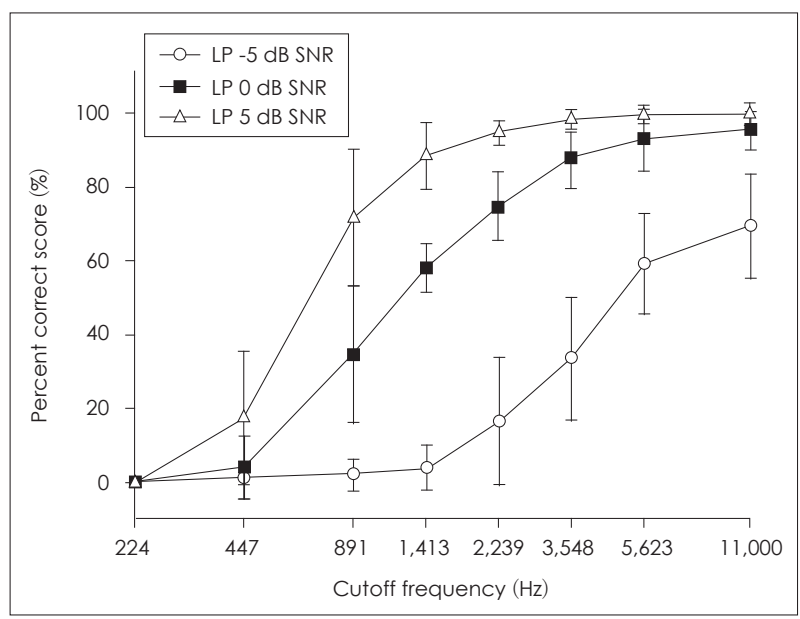

Fig. 1. Mean intelligibility scores and standard errors at different signal-to-noise ratios (SNRs) for the LP conditions. LP: low-pass filter.

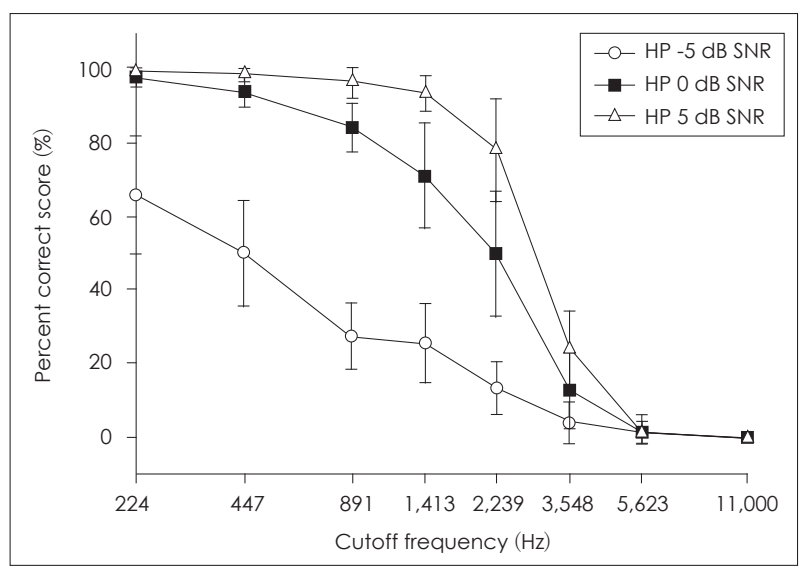

Fig. 2. Mean intelligibility scores and standard errors at different signal-to-noise ratios (SNRs) for the HP conditions. HP: high-pass filter. 
Table 1. The BIF for the KS-SL-A

\begin{tabular}{ccc}
\hline Band limit $(\mathrm{Hz})$ & CF $(\mathrm{Hz})$ & BIF $(\%)$ \\
\hline $224-447$ & 316 & 20 \\
$447-891$ & 631 & 16.2 \\
$891-1413$ & 1,122 & 10 \\
$1,413-2,239$ & 1,778 & 13.4 \\
$2,239-3,548$ & 2,818 & 16.9 \\
$3,548-5,623$ & 4,466 & 12.3 \\
$5,623-11,000$ & 7,864 & 11.2 \\
\hline
\end{tabular}

BIF: band-importance function, KS-SL-A: Korean standard sentence lists for adults, CF: center frequency

tween derived band-importance weights and obtained percent intelligibility values. Overall, band-importance weights were varied across frequency bands. The highest band-importance weight was $20.0 \%$ at the CF of $316 \mathrm{~Hz}$. The lowest band-importance weight was $10.0 \%$ at the CF of $1,122 \mathrm{~Hz}$. The importance of the frequency bands below the CF of $1,778 \mathrm{~Hz}$ was $59.6 \%$.

\section{Discussion}

The BIF for the KS-SL-A from this study was compared to the BIF for the K-HINT [6] in Fig. 3. The BIF for the KHINT was derived for 21 frequency bands; therefore, in order to compare the results from the 2 studies, cumulative raw data were used [4]. Overall, the cumulative band-importance weights for K-HINT were higher than the weights for KS-SLA below 3,000 Hz. In the case of the BIF for the KS-SL-A, frequency regions below $316 \mathrm{~Hz}$ and $2,818 \mathrm{~Hz}$ accounted for $20.0 \%$ and $76.5 \%$ of importance weight, respectively. The midpoint of the BIF was around at $1,122 \mathrm{~Hz}(46.2 \%)$. In the case of the BIF for K-HINT, frequency regions below $350 \mathrm{~Hz}$ and $2,900 \mathrm{~Hz}$ accounted for $22.9 \%$ and $78.8 \%$ of importance weight, respectively. The midpoint of the BIF was around at $1,170 \mathrm{~Hz}(52.1 \%)$.

The comparison of band-importance functions for Korean (current study), English [5] and Cantonese [4] sentences is shown in Fig. 4. The BIFs for English (9 frequency bands) and Cantonese (18 frequency bands) were also derived using different numbers of frequency bands. In order to compare the results from the 3 studies, cumulative raw data were used [4]. As shown in Fig. 4, low frequency regions below 1,100 $\mathrm{Hz}$ were more important in Korea and Cantonese BIFs compared to English BIF. In the case of Korea and Cantonese BIFs, frequency regions below 1,122 and $1,250 \mathrm{~Hz}$ accounted for $46.2 \%$ and $51.2 \%$ of importance weight, respectively. However, in the case of English BIF, frequency area below $1,160 \mathrm{~Hz}$ accounted for $34.8 \%$.

To obtain intelligibility data, the current study followed an

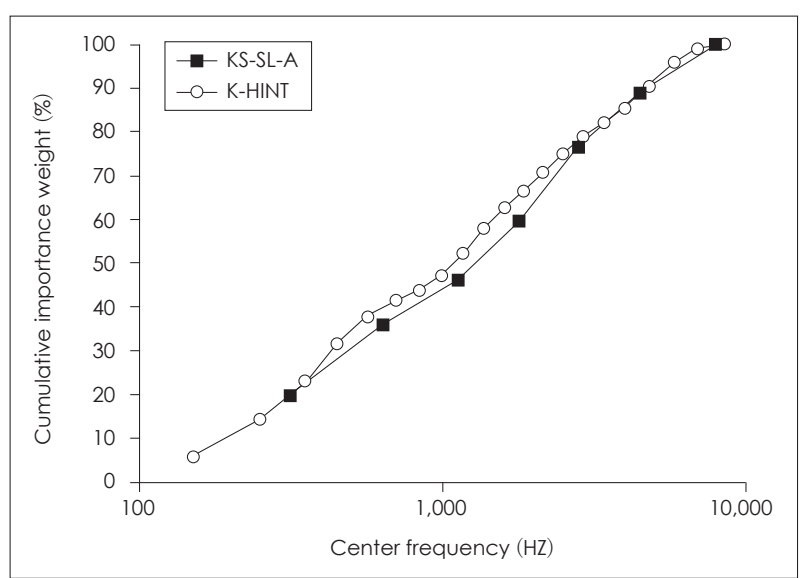

Fig. 3. Comparison of cumulative band-importance functions for KS-SL-A (square) and the K-HINT sentences (circle). The x-axis represents the center frequency and the $y$-axis represents the cumulative weight of importance. KS-SL-A: Korean standard sentence lists for adults, K-HINT: Korean hearing-in-noise test.

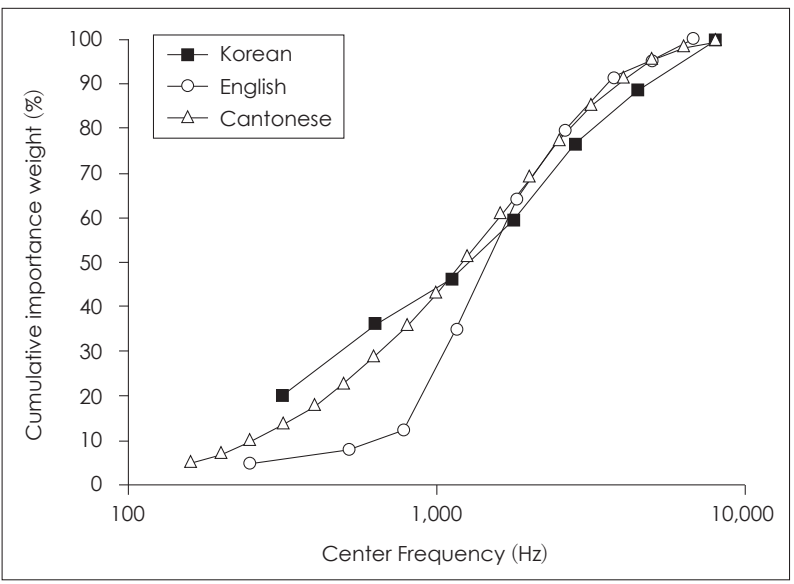

Fig. 4. Cumulative band-importance functions for Korean (square), English (circle), and Cantonese (triangle) sentences. The $\mathrm{x}$-axis represents the center frequency and the y-axis represents the cumulative weight of importance.

established procedure [13]. However, few recent reports used different methodologies to obtain intelligibility data $[16,17]$. For example, Warren, et al. [16] measured intelligibility using one-octave band-pass filters (CFs of 250, 500, 1,000, 2,000, 4,000 , and $8,000 \mathrm{~Hz}$ ) at five different speech levels in quiet. Healy, et al. [17] obtained intelligibility data using 21 bandpass filters from the $\mathrm{CF}$ of $150 \mathrm{~Hz}$ to the $\mathrm{CF}$ of $8,500 \mathrm{~Hz}$ at a fixed speech level (70 dB A) in quiet. While the current study obtained the intelligibility data using various high and low-pass filters at several SNRs, the two studies mentioned above used band-pass filters in a quiet condition. Because these studies used different methodologies, direct comparison may not be possible. Thus, validation studies may be required to know which method is more reliable to derive the BIF.

The present study had a few limitations. The BIF for the current study was derived using 7 frequency bands. Howev- 
er, other BIF studies derived their respective BIFs by using more segmented frequency bands such as bands 9 [5], 18 [4], and 21 [6]. Although the results of the present study provide accurate importance weights for the 7 frequency bands, further study is required to derive importance weights for more segments of frequency bands. Although we made comparisons with studies that derived their BIF with the same rationale, these studies used different methodologies such as different frequency bands and talkers [4-6]. Thus, an exact comparison across these BIFs may not have been achieved.

In addition, intelligibility performances in the present study were measured using the speech shaped noise which was used in previous studies [4-6]. However, intelligibility performances can be affected by different types of noise [18]. Thus, in order to identify whether differences in BIFs are evident across different types of noise, various types of noise like multi-talker babble may be considered in a future study.

The current findings can be applied for developing a hearing aid fitting formula for Korean listeners. By comparing to the formula for English listeners [3], one can take into consideration the finding of the importance of the low-frequency regions in Korean speaking and place more weight on lowfrequency gains when developing the hearing aid fitting formula for Korean listeners. Although other factors like dynamic range of speech should be considered to determine proper hearing aid gains, the present study may be an initial step to develop hearing aid fitting formula for Korean listeners.

\section{Acknowledgments}

This study was supported by a grant from Samsung Electronics Co., Ltd. and by Basic Science Research Program through the National Research Foundation of Korea (NRF) funded by the Ministry of Science, ICT \& Future Planning (NRF-2015R1C1A1A01052458).

\section{Conflicts of interest}

The authors have no financial conflicts of interest.

\section{REFERENCES}

1) American National Standards Institute. Methods for calculation of the speech intelligibility index. ANSI S3.5-1997 (R2007). New York, NY: American National Standards Institute; 1997.

2) Amlani AM, Punch JL, Ching TY. Methods and applications of the audibility index in hearing aid selection and fitting. Trends Amplif 2002;6:81-129.

3) Dillon H. NAL-NL1: a new prescriptive fitting procedure for nonlinear hearing aids. Hear J 1999;52:10-6.

4) Wong LL, Ho AH, Chua EW, Soli SD. Development of the Cantonese speech intelligibility index. J Acoust Soc Am 2007;121:2350-61.

5) DePaolis RA, Janota CP, Frank T. Frequency importance functions for words, sentences, and continuous discourse. J Speech Hear Res 1996;39:714-23.

6) Jin IK, Kates JM, Lee K, Arehart KH. Derivations of the band-importance function: a cross-procedure comparison. J Acoust Soc Am 2015;138:938-41.

7) Moon SK, Hee Kim S, Ah Mun H, Jung HK, Lee JH, Choung YH, et al. The Korean hearing in noise test. Int J Audiol 2008;47:375-6.

8) Hirsh IJ, Davis H, Silverman SR, Reynolds EG, Eldert E, Benson RW. Development of materials for speech audiometry. J Speech Hear Disord 1952;17:321-37.

9) Tillman TW, Carhart R. An Expanded Test for Speech Discrimination Using CNC Monosyllabic Words: Northwestern University Auditory Test No. 6. Technical report SAM-TR-66-55. Brooks Air Force Base, TX: USAF School of Aerospace Medicine; 1996.

10) Jang HS, Lee JH, Lim DH, Lee KW, Jeon AR, Jung EJ. Development of Korean Standard Sentence Lists for Sentence Recognition Tests. Audiology 2008;4;161-77.

11) Sims DG. The validation of the CID everyday sentence test for use with the severely hearing impaired. J Acad Rehabil Audiol 1975;8: $16-22$.

12) American National Standard Institute. Specification for audiometers. ANSI S3.6-2010. New York, NY: American National Standards Institute; 2010.

13) Studebaker GA, Sherbecoe RL. Frequency-importance and transfer functions for recorded CID W-22 word lists. J Speech Hear Res 1991;34:427-38.

14) Fletcher H, Galt RH. The perception of speech and its relation to telephony. J Acoust Soc Am 1950;22:89-151.

15) Kim GG, Jin IK. Measurements and applications of the speech Intelligibility Index (SII). Audiol Speech Res 2015;11:186-95.

16) Warren RM, Bashford JA, Lenz PW. An alternative to the computational Speech Intelligibility Index estimates: direct measurement of rectangular passband intelligibilities. J Exp Psychol Hum Percept Perform 2011;37:296-302.

17) Healy EW, Yoho SE, Apoux F. Band importance for sentences and words reexamined. J Acoust Soc Am 2013;133:463-73.

18) Vokál J, Dlouhá O. The sentence intelligibility in different types of noise regarding people with a normal hearing ability. Prague Med Rep 2009;110:60-6. 